\title{
Transforming the Kindergarten Experience by Design: A Comparative Research Case Study
}

\author{
Raechel French ${ }^{1}$, Lennie Scott-Webber ${ }^{2} \&$ Anjana Sivakumar ${ }^{3}$ \\ ${ }^{1}$ DLR Group, K12 Education Planner, Austin, TX, USA \\ ${ }^{2}$ AIA Affiliate, Estero, FL, USA \\ ${ }^{3}$ DLR Group, LEED Green Associate, Architectural Designer, Austin, TX, USA \\ Corresponding author, Lennie Scott-Webber, INSYNC: Education Research + Design, 14223 Wild Timber \\ Court, Estero, FL, 33928, USA. E-mail: lenniesw.insync@yahoo.com
}

Received: December 10, 2019 Accepted: January 18, 2020 Online Published: February 17, 2020

doi:10.5539/jel.v9n2p82 URL: https://doi.org/10.5539/jel.v9n2p82

\begin{abstract}
Kindergarten education is becoming a priority throughout the USA as research shows its importance on later in life outcomes. The State of Washington instated full-day kindergarten for all public-school students beginning in 2016-2017. It “...is part of the state's constitutionally protected definition of 'Basic Education'” (Reykdal, n.d., n.p.), working to support all children in the state. Acting on this new state requirement, one school district chose to design and build a center dedicated solely to kindergarten education, housing approximately 600 kindergarteners. The school was divided into four 'neighborhood pods' each with immediate access to specific activity programs (i.e., dining, interventionists, elective functions) reflecting a new 'expanded push-in' model and reduce transition times. This model was compared with a 'traditionally' operated kindergarten where learners travel to activity programs throughout a campus (i.e., dining, interventionists, electives). A human-centered research design using mixed-methods for this comparison study between an 'expanded push-in' and a 'traditional' model was used to understand the impact of this new architectural solution particularly focused on timing transitions between the classroom and activity program settings. Findings discovered a reduction in the length of transitions between accessing the programs by recaptured seven school days of learning time (approximately 45 hours), when compared to the traditional one, and more positive connections between students/students, teachers/teachers, and students/teachers to build community.
\end{abstract}

Keywords: design, education, post-occupancy, case study, kindergarten, ethnographic research

\section{Introduction}

Evidence suggests a kindergarten experience is a critical path for all children. "Students who attend school from kindergarten through secondary school typically spend more that 13,000 hours of the developing brain's time in the presence of teachers" (Desautels, 2016). Clearly, kindergarten is important, but putting 600 students in one building is unusual and perhaps unprecedented for this level of cohort. This situation necessitated a new design vision to support the state's new mandate.

The vision for the design team began by first understanding why 'it' (a kindergarten center to host 600 children) was needed. And then, and perhaps more importantly, how it would function for all of these children along with the host of educators and staff who would be working here. The design team also spent time at different kindergarten sites throughout the district to understand first-hand how learning was currently experienced.

The new design vision resulted in the development of community bringing all of the typically spread out activity program areas into what is now referenced as 'neighborhood pods.' This vision was to support the building of community and facilitate less time for students transitioning from their classroom to the activity programs (i.e., gym, or dining, or playground, or other specific activity program areas). A design hypothesis was generated suggesting a change from a more 'traditional' model (students moving across a campus to access activity programs) to this 'expanded push-in' model (activity programs accessed within a neighborhood) would amount to approximately 15 extra days of learning without having to add any extra days to the school calendar. Once the building was built and 'lived in' for almost one year of occupancy, the architectural firm's K12 education design team decided to vet their design hypothesis using a case study research method. The comparative research case 
study is shared here.

This paper will provide a background of kindergarten learning years, a brief overview of the design's vision and process, and its early understandings from both Case \#1 and Case \#2, along with the design's achievements. Then, research design protocols, research questions, methods for how the study was conducted, and discussions of the findings are shared. Limitations and Conclusions finalize the article.

\section{Background-Kindergarten Years}

It has been well documented that early childhood learning is critical for a life of learning and positive human development. "The first five years of a child's life are fundamentally important. They are the foundation that shapes children's future health, happiness, growth, development and learning achievement at school, in the family and community, and in life in general" (Anonymous, n.d.). The kindergarten experience must build on this early learning one, and it is usually for children ages five and six. "It gives children the opportunity to grow and develop through play, which is considered the best way to learn. It is also a time for children to expand their love of learning, build knowledge, develop their ability to get along with others, and explore ways of reaching out to the world" (Taylor, 2011).

The focus for this kindergarten cohort is on: (a) stimulating the brain, (b) developing social skills, and (c) addressing and increasing awareness of health and wellness both at the physical and emotional levels. It continues the same goals as did the early learning education phase of pre-school, and prepares these young learners for the transition into elementary school. The pedagogy, or teaching practices for these kindergarten places follows the idea of activity-based, or 'circuit' learning in activity zones in the formal learning place (aka 'the classroom'). These activity zones are designed to directly support the types of learning experiences the pedagogy of this age cohort requires. All manners of simple learning tasks are taught from how to line up, to get one's own items out of a personal cubby, and to tying one's shoes, etc. So, sight lines for a teacher are very important as staying on task at this early age is not easy for little ones. Transparency and view planes to the outside and nature along with the ability to see into the corridors or transition areas are important considerations for safety and security.

Taylor (2011) says,

"I'm often baffled when I talk to those who think a kindergarten class, or any class for that matter, should be quiet. For one thing, students at this age love to talk. Kindergartners' vocabulary is growing very rapidly, and many are developing the power to express ideas. When we provide students with opportunities to talk about what they are learning, we are helping them to construct meaning and learn from their experiences. Children should be working together, problem-solving and sharing ideas and activities. This helps children learn how to think through problems and find solutions together. In addition, the teacher should be reading to students throughout the day. One of the most important things teachers can do in preparing children for success in school and in reading is to read aloud to them. Read-alouds help build listening skills, comprehension skills, and so much more. At times the children should also be reading, to themselves, other students, or the teacher" (n.p.).

The teacher often uses the areas outside of the classroom for multiple purposes including breakout activities, or push-out segments for special support, art, eating, and moving from one area to the other. The architecture of the place including interior design affordances (i.e., furnishings and fit-outs) must support multiple postural changes, and technologies both analogue (non-digital) and digital, and lots of storage; the latter is critical.

\section{Design Understandings and Solution Development}

Faced with a state mandate to instate full-day kindergarten and accommodate growing capacity demands, for this school district it established its first kindergarten center at a selected site for this elementary building. As mentioned, this design team spent time at multiple kindergarten sites to more clearly understand and experience the learning and teaching needs for this age cohort in what might be referred to as typical 'traditional settings.' Early insights revealed: (a) a lot of rules and regulations, (b) creativity seemed stifled, (c) special program activity centers were spread out across a campus, and (d) transitioning from one program activity center to another took a lot of time. With actvity programs and specialty areas spread out across a campus in this traditional model, it meant that the design of the school did not appear to revolve around the student, was hard to generate a sense of community, and transition times to these specialized activity areas were quite substantial. Time spent observing at a traditional kindergarten showed that teacher coordination and the length of transition times were impacting learning time (see Figure 1). 


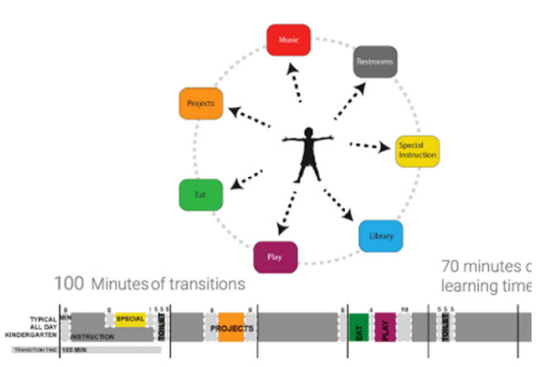

Design Hypothesis

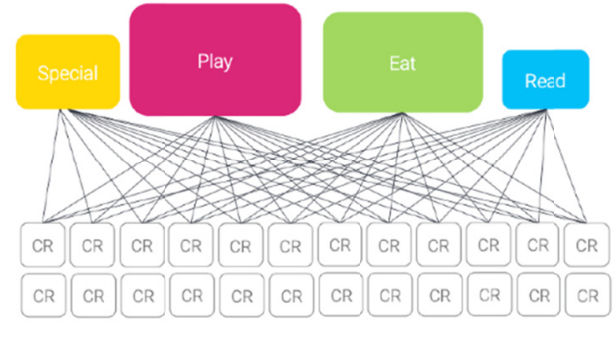

Existing Condition

Figure 1. A traditional, more non-student-centered model

Source: French, Scott-Webber, Ferking, \& Fulton, 2015.

A new vision meant the larger architectural programs requiring more space, such activities as dining and project areas, would be broken down into smaller spaces and integrated into what was termed 'pod neighborhoods.' Other qualities not studied here but indicated in the architectural firm's project brief included: "Given its significant effect on learning, indoor environmental quality received diligent design attention, including temperature, air quality, daylighting, and acoustics. The kindergarten students spend a lot of time learning on the floor. Thus, radiant floors sourced from a geothermal ground-source heat pump provided warmth design created (approx. 65,000 sq. ft.) spaces to support early learning, while maximizing the potential synergies of age-specific students" (Anonymous, n.d.).

The new vision referred to here as an 'expanded push-in' design concept of the Case \#1's Kindergarten Center took its inspiration from the form of the ancient Nautilus shell (see Figure 2). The shell represents an architectural symbol of proportional perfection. It is also a symbol for expansion and renewal as it grows increasingly larger chambers while shedding old ones throughout its life. This design solution focused on developing neighborhood as 'pods' (e.g., a group of 6 individual 'teacher owned' classrooms with associated pod spots and dining tables). The smaller 'pod spots' (e.g., a small table with 4 to 6 chairs with analogue tools for the specialists to work with students) were located directly outside of each classroom. This design was intended to enable the 'pod spots' within each pod neighborhood to be incorporated into a more inclusive learning place for students, interventionist staff, elective staff and teachers. Building community and a sense of belonging allows all to become more engaged and have a higher sense of wellbeing. A design hypothesis developed: Will this new design concept result in measurable results relative to (a) a gain of learning time as a result of reducing transition times, and (b) a real sense of building of a community (see Figure 2). The external and internal designs ideations flush out this vision's concept (see Figure 3).
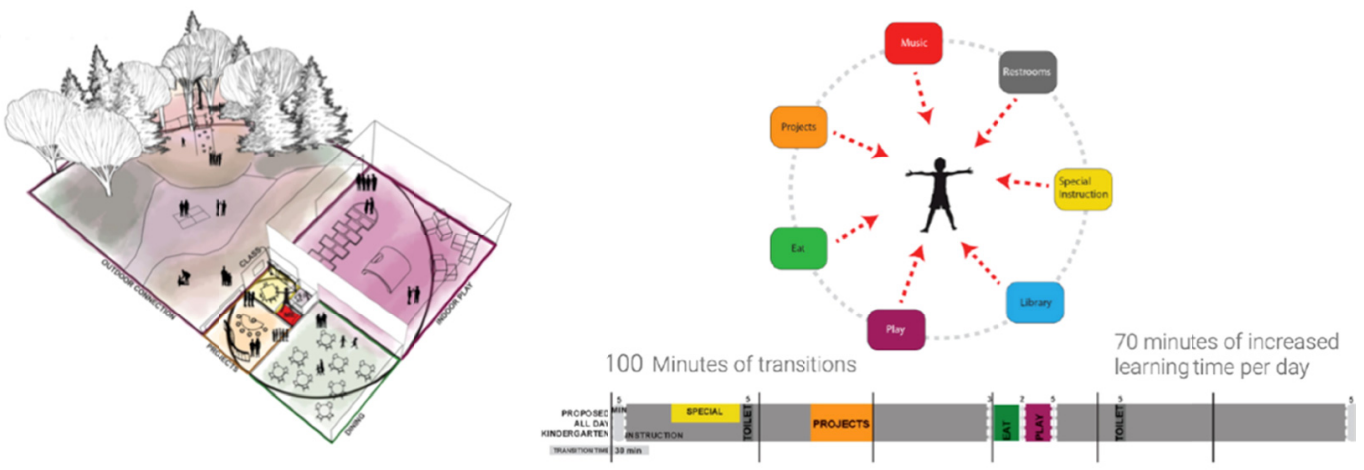

Figure 2. Design from the ancient nautilus shell—perfect proportions/and re-centering with a student-focus Source: French, J. et al., 2015. 

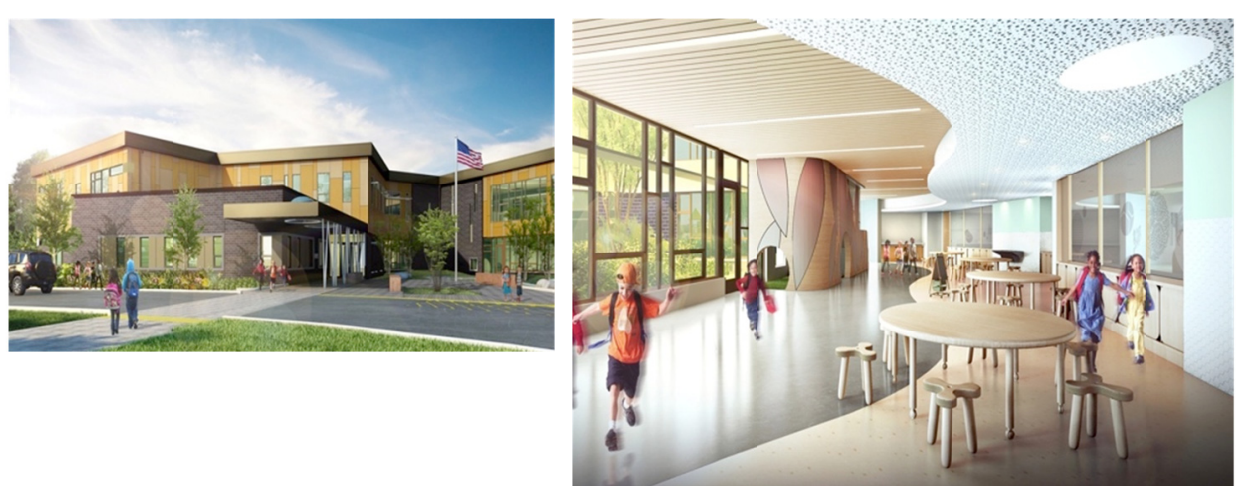

Figure 3. Design vision for exterior and interior

Source: French, J. et al., 2015.

The new vision focused on a design solution that would functionally maximize learning time. The school's architectural program was redefined to eliminate 'learning time lost' to transition travel. A concept evolved where teachers and specialists would still have their respective owned classrooms but also intentionally designed adjacent spaces, now referred to as 'pod spots' - to allow students to stay in their respective 'pod neighborhood.' A before and after activity mapping plan was generated to test the idea, along with a full floor plan sketch of how these activity programs would fit into the nautilus proportions (see Figure 4). To further a sense of community and support wayfinding, items of nature were called upon to ensure proper identification (see Figure 5).

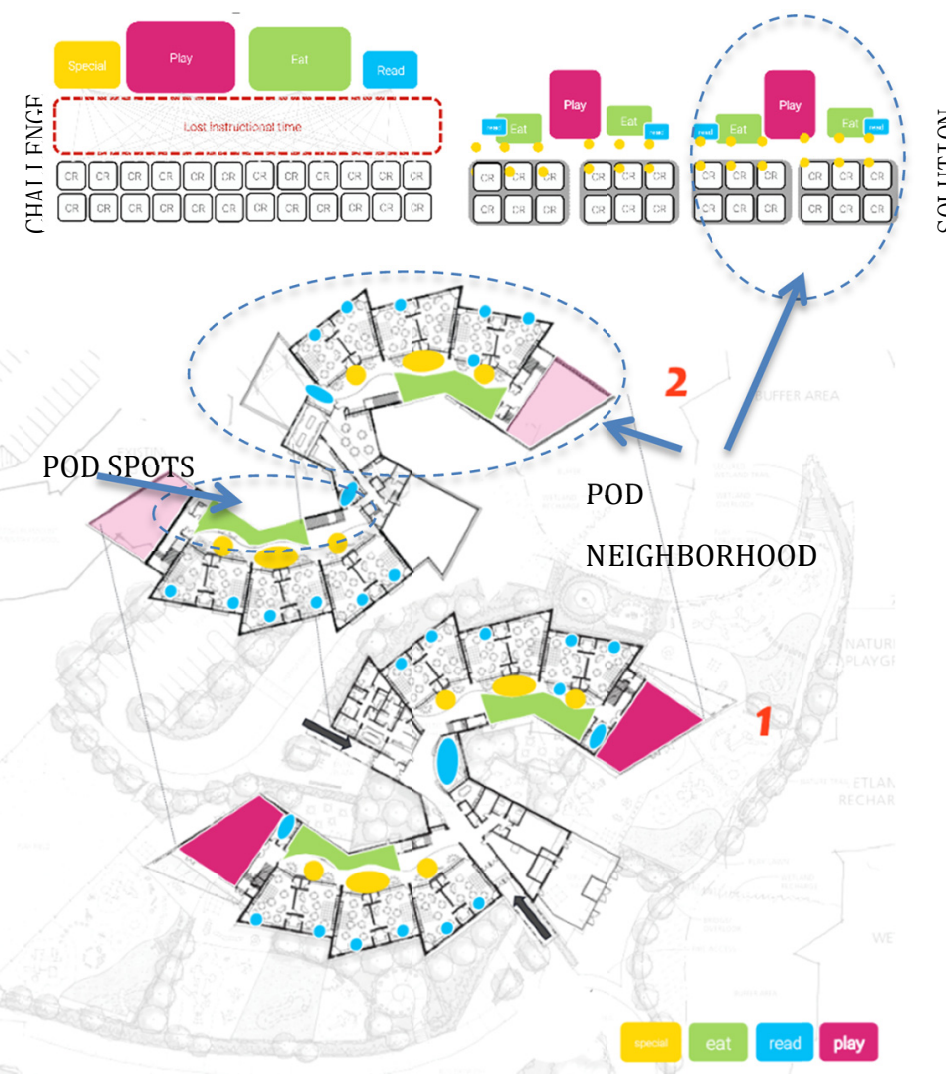

Figure 4. Ideation—-before and after/sketches for the new design (floors $1 \& 2$ ) 


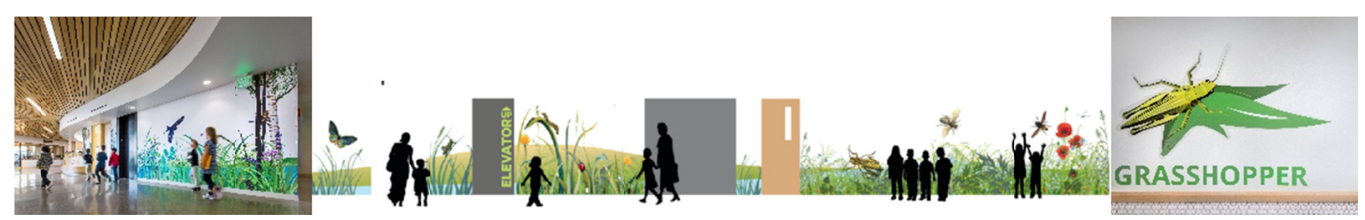

Figure 5. Wayfinding, community building using nature as inspiration

Source: French et al., 2015.

The re-centering of focus upon the student produced a design hypothesis that this expanded push-in design model could potentially reduce transition times and thus gain extra teaching and learning days without physically adding more days to the yearly calendar. A Comparative Research Case Study post-occupancy method was used to test the design hypothesis. The research method and study's findings are presented next.

\section{Method}

Members of the architectural firm's K12 Education Practice research team (not the designers involved), along with an external research consultant conducted the research. A major goal of the study was to ensure scientific rigor within a real-world project setting.

"The measure of good research often is associated with a rigorously scientific process, and this is justified for many areas of inquiry...Typically, the scientific method involves a process of identifying a problem or question, forming a hypothesis, testing the hypothesis by conducting an experiment or study with proper controls, checking and interpreting results, and communication" (Hanington, 2010, p. 19). For this study, the Human-Centered Research Design protocol (HcRD) (Scott-Webber, 2011) was utilized in post-occupancy to assess the impact of the design of the built environment on student and educator behaviors.

\subsection{Human-Centered Research Design Protocol}

The focus for human-centered research is grounded in Environment Behavior Psychology and works to understand how the design of the built environment impacts human behavior. Multiple methods are often used to answer this question, but fall into two primary areas - qualitative and quantitative. To ensure a rich data set is generated, the HcRD protocol always uses a mixed-method (Johnson, Onwuegbuzie, \& Turner, 2007) research design (inclusive of both quantitative and qualitative) for comparative purposes, to limit research bias, and expand and strengthen the studies' conclusions. This HCRD method uses the following:

- Quantitative techniques = literature review, "questionnaires and surveys", and quantifying qualitative items, and

- Qualitative techniques = "observing and talking to people...these methods are typically ethnographic (Zeisel, 1984) in nature, and may include participant observation, artifact analysis, photo and diary studies, contextual inquiry, cultural probes, and other methods designed to sample human experience" (Hanington, 2010, p. 23).

The designers on this project understood clearly the need to gather systematic information as, "Designers are equally accountable for the conduct of good quality research and, in particular, for being systematic in their approach to information collection, recording, synthesis, and analysis" (Hanington, 2010, p. 23) (see Figure 6). 


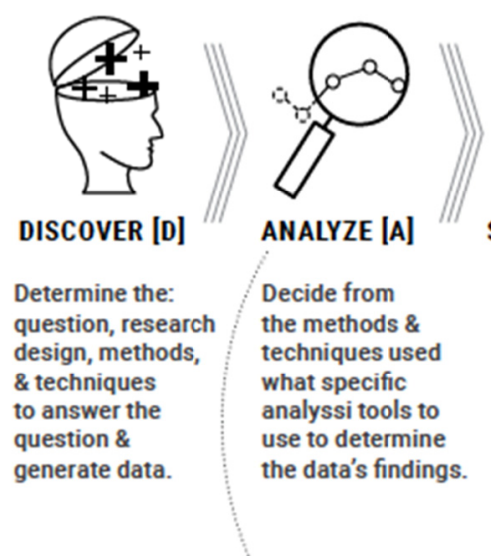

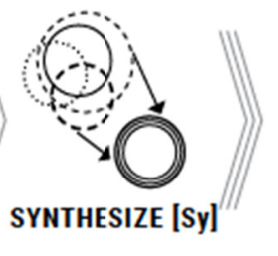
Take the infor- mation generated from the multiple analytic methods \& develop an under- standing of what the findings mean

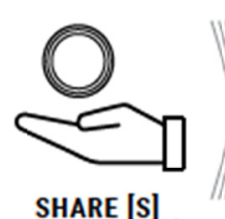

Provide multiple types of items for sharing to multiple user groups: client, designer, conference presentation, peer-reviewed journal article...

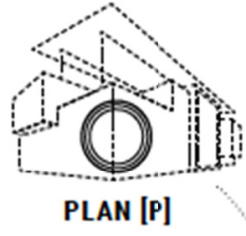

Use the "truth" from the findings to revisit the building, and/or determine what items should be incorporated into the next design.

Figure 6. A Human-centered research design protocol (HcRD) (image with permission of DLR Group)

The research design and human subjects' protocol was sent to a third-party institutional review board for research approval, shared with the schools, and then both schools' submitted voluntary consent forms back to the research team. Once consent forms were received the research was scheduled to commence.

This comparative study used two schools for convenience samples, with two 'typical' groups of students to study, in one age cohort, and a minimum of at least two researchers observing during a two-day timeframe, post-occupancy and late in the school year (May). One school was considered the 'new' school and one 'traditional' for comparative purposes. The focus was on transition activities. The researchers reviewed Environment Behavioral ethnographic techniques as developed by Zeisel (1984). The process employed pre-planning the following: (a) semi-structured set of research-guided questions to be used for interviews with administration and educational staff members, (b) two days of on-site behavioral observations focused on timing movement activities through space along with the direction of that travel, and (c) the collection of photographic traces at identified timed intervals. These observations were recorded into Excel spreadsheets and analyzed to generate a quantitative output comparing these two models. Next are the research questions.

\subsection{Research Questions}

The following research questions were addressed in this study:

- RQ.1. Does the new 'expanded push-in' design, bringing dining and other activity spaces into a 'neighborhood pod' reduce overall transition times and increase time spent on meaningful learning and play?

- RQ.2. How might the kindergarten learners and teachers utilize the new pod spot designs?

- RQ.3. What other impacts does a new pod neighborhood design have on kindergarten learners, educators, and school staff?

To lessen bias, data collection techniques were analyzed separately by each individual researcher and then compared. Observations and traces were coded, according to Zeisel's (1984) method for coding specific behaviors, and then the data combined both quantitative and qualitative to generate the findings. Two other individuals worked on the analysis of the data. A granular view of the design research process is shared next.

\subsection{Observation Techniques Detailed}

Two sites in the new design for Case \#1 were used for gathering the data, the neighborhood pods of 'Caterpillar' and 'Grasshopper.' Only two pod neighborhoods were studied as all are designed the same (refer back to Figure 4). The only difference was one neighborhood was located on the first floor and one on the second for research variety. Behavioral observations were pre-coded so each observer would be able to annotate (make quick notes) on the floor plans and thus allow for the data to be collected in the same way to ensure inter-rater reliability. Photographic traces were taken every 15 minutes from specific vantage points. The diagram below shows the Grasshopper pod neighborhood with its pod spot areas, and the researcher's annotations (see Figure 7). Both researchers observed each segment of the building over a two-day time frame. Observers switched floors mid-day to avoid bias in data recording. 


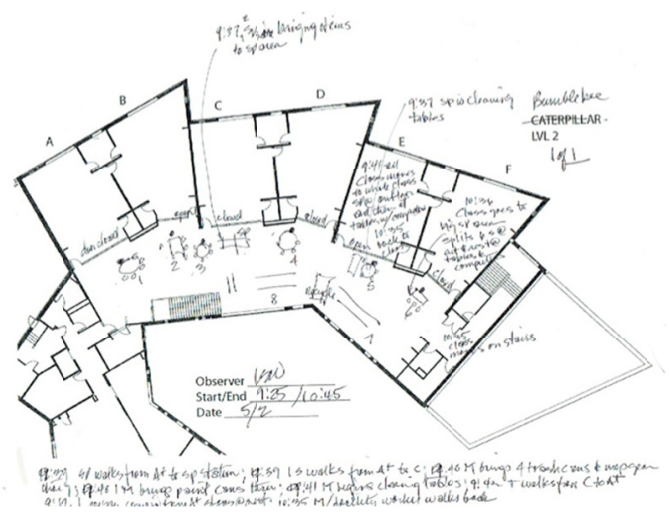

Figure 7. Annotated floor plan of observed behaviors

The researchers looked for patterns and recorded the times for: (a) the transitions from one program activity to the next, (b) use of pod spots, (c) who used them, and (d) overall activities within the pod's neighborhood. Below is an example of an early round of a data sheet, and then the pattern of travel through the neighborhood (see Figure 8). These annotations were put into spreadsheets to begin the pattern analysis, scrubbed and reviewed by the researchers. Then, the physical patterns were reviewed to understand how people were traveling through the neighborhood (see Figure 9).

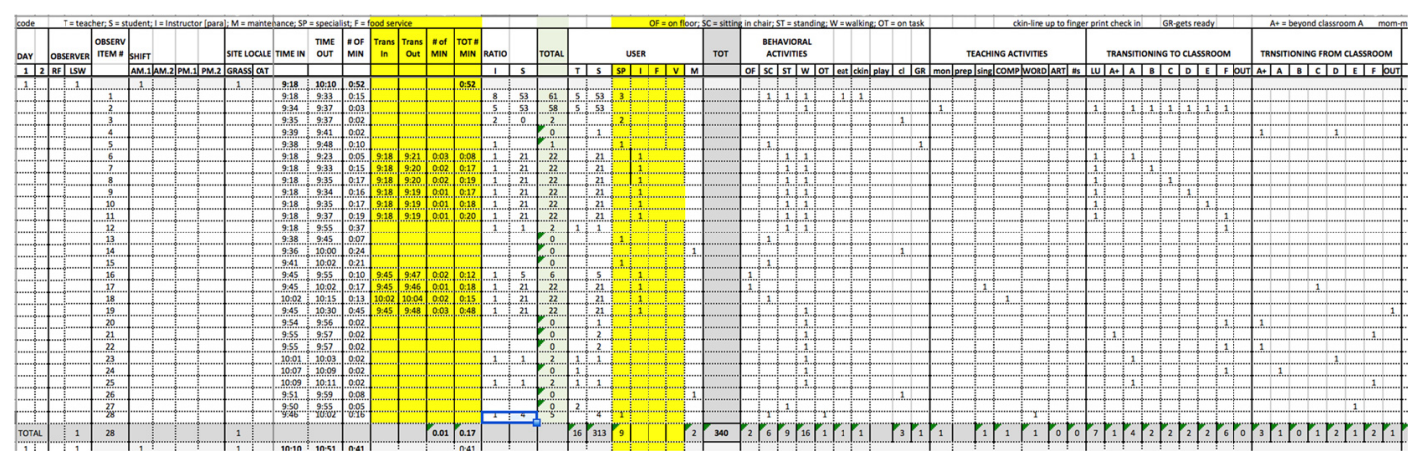

Figure 8. Early round of data input into spreadsheet

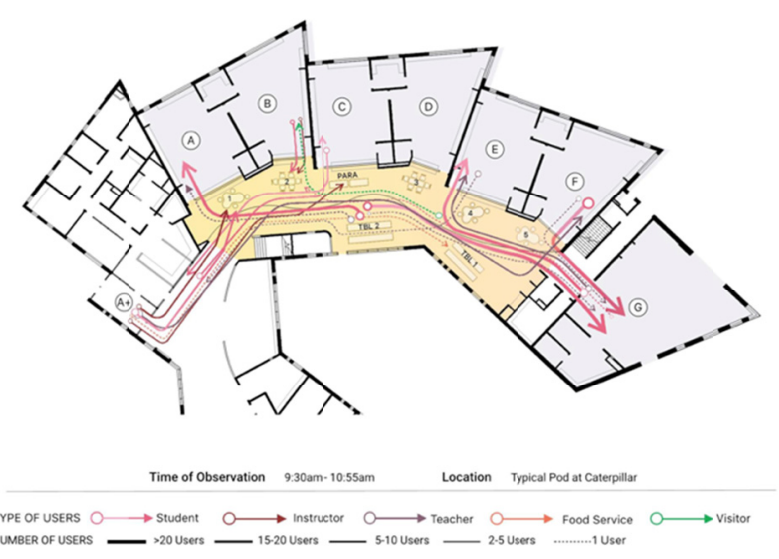

Figure 9. Mapping paths of travel within one pod 


\subsubsection{Photographic Traces}

Photographic traces from a site reveal 'traces' of activities by the way in which physical items in a space are left behind. An example of this idea is when sidewalks are placed on a campus to direct the path of travel. Yet, on observation the footprints of travel through the grass leaves a 'trace' that perhaps a more convenient line of travel should have been planned. Pattern traces then are used to confirm observations and verify. For the two days on site, each researcher recorded the pod's neighborhood traces. For analysis purposes, the team adapted Zeisel's (1984) method and coded for these five traces generating activity behavioral codes: (1) adaptations of use, (2) displays of self, (3) by-products of use/i.e., identification and wayfinding, and (4) public messaging. Each photograph was coded for activities based on behavioral trace codes, and then all codes were transcribed into a spreadsheet once again for qualitative analysis purposes (see Figure 10). In an effort to verify the findings of this new design, a second case study was done on a 'traditionally' designed kindergarten. That information follows.

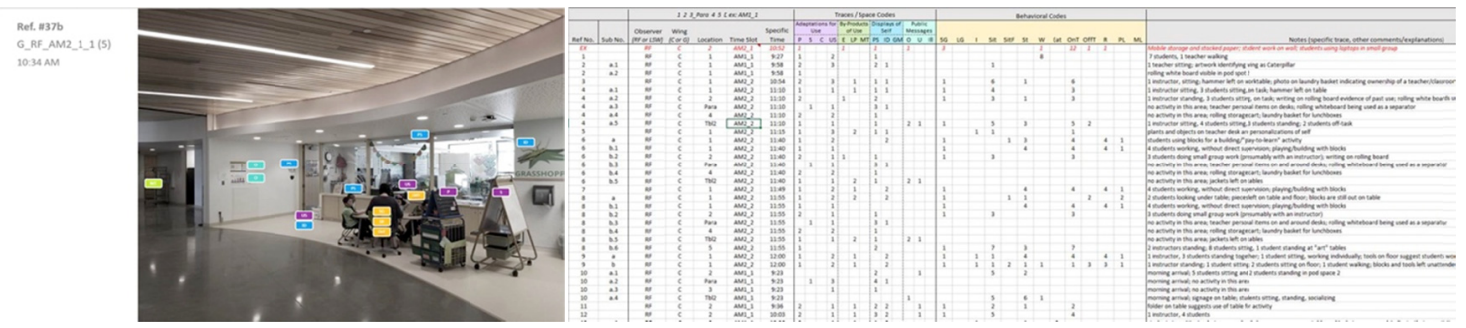

Figure 10. Photographic trace annotation/example spreadsheet

\subsubsection{Comparative Case Study—A Traditional Model}

In order to verify the findings from the new 'expanded push-in' design study, the research team conducted a comparative case study at a 'traditionally' designed elementary school having a kindergarten area in the same school district for our Case \#2. This time there were three researchers observing the two kindergarten classrooms, (as researchers would have to follow the student to know where they were going as distances were longer), with one researcher assigned to each classroom group and one researcher assigned to any outliers, or students who moved or acted separately from the whole class. The research objectives were the same and techniques executed followed the original research protocols for the first study of the new model. Observers again recorded student behavior and transitions over a two-day period late in the year, post-occupancy (May). The Case \#2's campus was selected for the key differences and parallels that could be used to test it against the design for Case \#1's Kindergarten Center.

\section{Findings}

\subsection{Key Differences \& a Parallel}

Case \#2-Some of the key differences were:

- Design was the more 'traditional' school model, with dining, recess, and special activity program areas spread out across the campus.

- Classrooms were arranged as a 'cul-de-sac', with all the classrooms sharing a collective 'pod spot area,' which was informally divided into two learning areas and one area for para-educators.

- Each 'cul-de-sac' was comprised of mixed-level age cohorts. The one that was observed contained two kindergarten classes, two first-grade classes, and a third-grade class.

- Classrooms had no glass for transparency on their interior walls, providing no opportunity for passive supervision into the pod spots.

One key parallel at Case \#2 showed the:

- Classrooms were part of a neighborhood, with pull-out or 'pod spots' immediately available outside of their classrooms, all at the end of a cul-de-sac.

At both campuses, transition times were grouped into and analyzed by:

- Overall transition time 
- Transitions to/from dining

- Transition to/from recess

- Transition to/from specials

When comparing whole class transitions of the two schools, the design of Case \#1 appears to save a total of seven school days ( 45 hours) in transition travel time over the year. This figure is less than the design hypothesis of 15 days, but significant never the less. This final figure was calculated by averaging the six whole class transitions that happen during the school day (same schedule for both schools). These transitions included six 'instances' including: (a) classroom-to-recess, (b) recess-to-classroom, (c) classrooms-to-dining, (d) dining-to-recess, (e) classrooms-to-specialists, and (f) specialists-to-classroom. At times, the path of travel varied between each instance. The following plans show the different paths taken, the averaged transition times, and the distances traveled for each of the transition types, comparing Case \#1 and Case \#2 (see Figures 11 and 12). Since specialists' programs varied daily and had different locations, all were accounted for together with distances traveled and transition times averaged across all instances (see Table 1).
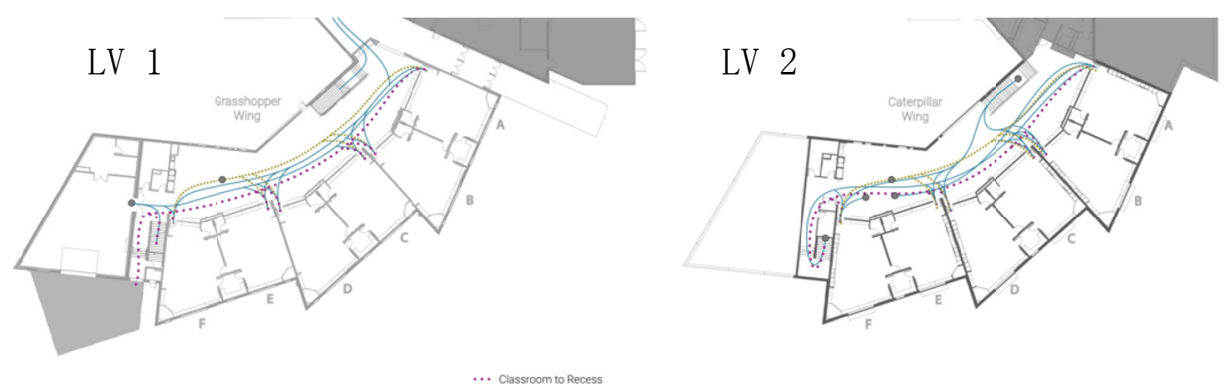

Figure 11. Whole class transitions for Case \#1, floors 1 and 2

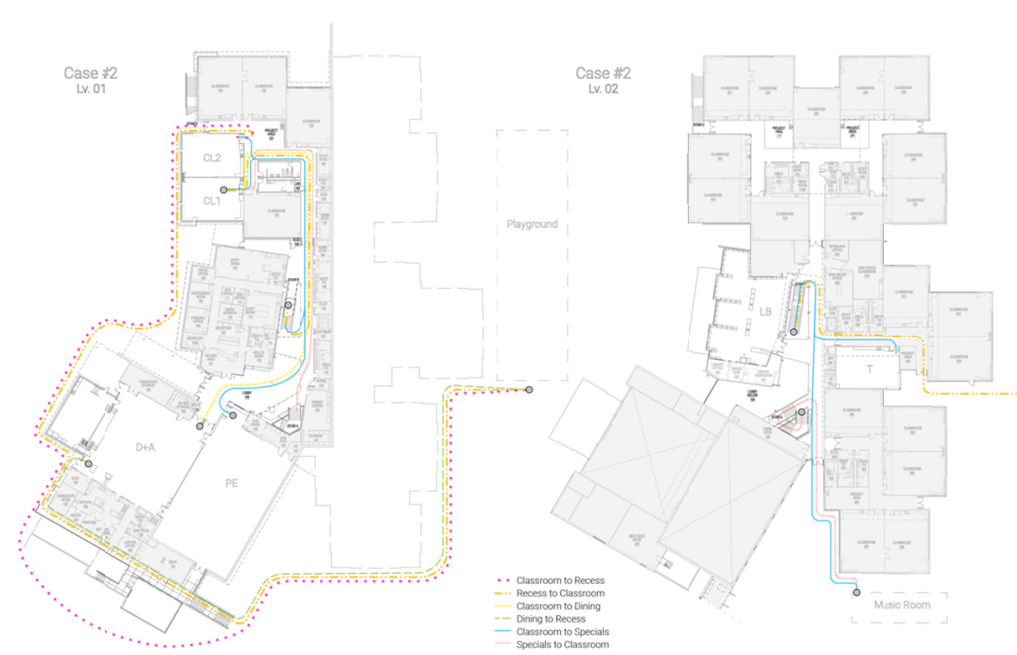

Figure 12. Whole class transitions for Case \#2

Table 1. Comparative average daily transition times $(*$ not established in the data collection)

\begin{tabular}{lll}
\hline \multirow{2}{*}{ To/From } & \multicolumn{2}{c}{ Average Daily Transition Times } \\
\cline { 2 - 3 } & Case \#1 & Case \#2 \\
\hline Classrooms to/from Recess & $03: 00$ & $08: 34$ \\
Classrooms to Dining & $01: 40$ & $02: 26$ \\
Dining to Recess & $0 *$ & $06: 13$ \\
Classrooms to/from Specials & $03: 42$ & $09: 23$ \\
Total Transition Time & $10: 02$ & $26: 36$ \\
\hline
\end{tabular}


One can see that the transitions for Case \#1 are shorter and simpler, with locations close by and specials consolidated. Studies of the various transition paths taken in both schools revealed that the average travel distances in Case \#1 were $1 / 10^{\text {th }}$ of the average travel distances in Case \#2 (see Table 2).

Table 2. Comparative average daily transition times

\begin{tabular}{llll}
\hline & \multicolumn{3}{l}{ Average Travel Distances (ft.) } \\
\cline { 2 - 4 } To/From & Case \#1 & Case \#2 & Case \#1/Case \#2 \\
\hline Classrooms to/from Recess & 109 & 1056 & $10 \%$ \\
Classrooms to Dining & 42 & 449 & $9 \%$ \\
Classrooms to/from Specials & 73 & 558 & $12 \%$ \\
\hline
\end{tabular}

It's important to note that transition travel time does relate directly to walking speed. The times logged in these observations for both schools included intermediate stops to ensure the entire class stayed together, or for teachers to attend to any disruptive behavior. The longer and more complicated a travel path, including going up or down stairs, the more intermittent stops were required, extending the transition travel time even more. The design hypothesis was the longer the transition time the more 'lost learning time.' However, several teachers and specialists anecdotally indicated that particularly at the beginning of a school year, all time is learning time. Each child is learning basic tasks and skills-lining up, walking as a group, and staying focused - in addition to academic skills.

\section{Discussion by Research Question from Observations Analysis}

6.1 RQ.1. Does the New 'Expanded Push-in' Design, Bringing Dining and Other Activity Spaces into a 'Neighborhood Pod' Reduce Overall Transition Times and Increase Time Spent on Meaningful Learning and Play?

Transition travel times, at Case \#1, from one program activity pod spot to the next within the neighborhood averaged 35 seconds, while the whole classroom transitions from door of classroom to specials or to the gym was three minutes. Teachers at Case \#1's location felt the benefits of the reduced transition time in the daily schedule as reclaimed learning time.

"We're able to maximize the school day because everything is so close together...(at) my old school, we'd have to allow at least five minutes for transitions and here...it's less than a minute"-Teacher.

\subsection{RQ.2. How Do the Kindergarten Learners and Teachers Utilizing the New Pod Spot Designs?}

At both Case \#1 and Case \#2, pod spot areas were located immediately or almost immediately outside the classroom; as such, transition time to or from them were negligible for both campuses. It should also be noted that the size, quantity of pod spots, and arrangement of classrooms varied greatly between the two, resulting in a different degree of utilization.

\subsubsection{At Case \#1}

Note that each neighborhood at this school had six classrooms, all with adjacent pod spot spaces designed immediately outside the classroom intended for that classroom educator's use for specialists. While specialists frequently utilize the push-in method, by which this person works with groups of students within an area in the classroom itself, the pod spot spaces outside 'acted like an extension of the classroom.' Long tables for mixed-use of dining and other hands-on activities were provided in the expanded hall space. On site the researchers coded these pod spots as: 1, 2, para, 3, 4, 5, TBL 1, and TBL 2 (see Figure 13). The two floors observed at Case \#1 utilized the pod spots differently. 

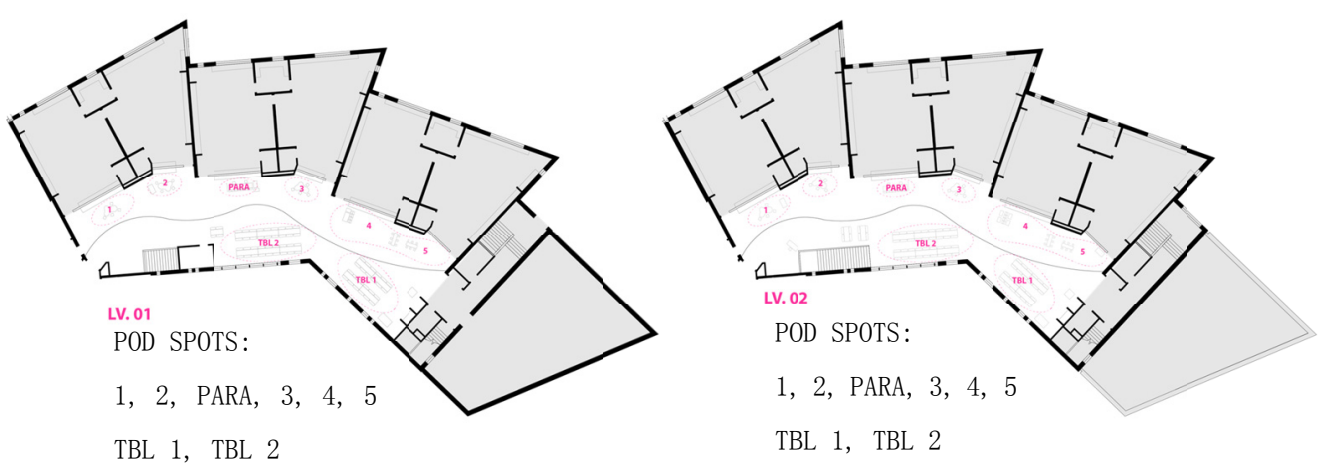

Figure 13. Floor plans with labeled pod spots for Case \#1

Analysis disclosed intended use and unintended usage:

Intended use:

- Small group activities with specialists took place at the pod spots 1,2 and 3 rotating in/out of their classroom to work with an interventionist, a 'push-out scenario'

- Pod spot spaces outside the classroom 'acted like an extension of the classroom'

- Classrooms continued the use of having specialists frequently utilize the push-in method, by which this person works with groups of students within an area in the classroom itself

- Small group activities moved out to the pod spots allowing interventionists/specialists to have more room for a variety of activities (see Figure 14).
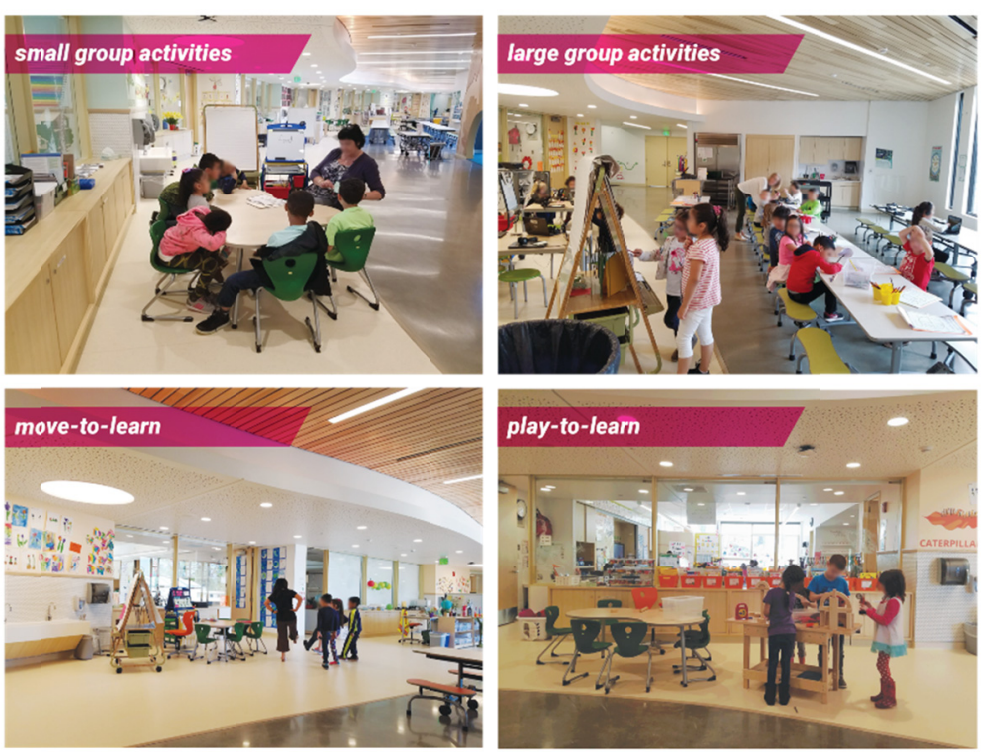

Figure 14. Examples of pod space use for Case \#1

Unintended use:

- One classroom had a set of desks for paras/specialists and nothing for students

- $\quad$ Small group activities took place at TBL 1 and TBL2

- Two classrooms' pod spots now formed a combined 'specialist's area' for activities such as technology, art, or music; utilizing all of pod spots 4 and 5, and the dining table areas (TBL 1 \& TBL 2 for both 
floors).

Analysis submits perhaps a mitigation of the potential use for the classroom educator with this repurposing of three of the six intended pod spot usage. Furthermore, when activities were scheduled in the specialist's area it would be utilized for approximately three hours and twenty-five minutes concurrently. Several quotes from educator's and staff support the information shared above.

During interviews, the following comments were made about pod spot usage:

- "I really like being part of the classroom...but there's just not room with the play-to-learn and everything going on in there" (specialist)

- "...even though the kids are being 'pulled-out' of the classroom into the pod spots...that works well" (teacher)

- "She (meaning the interventionist/specialist) started in our classroom...but...she liked it out in the hall (pod spot). She's right there...even though there's a window between us, I still feel like she's part of the room" (teacher)

- "The stigma of pulled-out activities is changed with the direct classroom connection" (teacher).

The various pod spaces in Caterpillar (second floor) were collectively used for a total of 8 hours and 40 minutes over the course of the observation timeframe. Meanwhile, the various pod spaces in Grasshopper (first floor) were collectively used for 6 hours and 11 minutes -2 hours and 30 minutes less than in Caterpillar. Assuming 6.5 hours in each school day, even the most frequently used pod space (area 2 in Caterpillar) was only utilized for approximately $27 \%$ of the day. The table below outlines the total amount of time spent in small groups during each day of observation at each table area for each floor observed (see Table 3).

Table 3. Total time utilized for small group at Case $\# 1$-across two days of observation

\begin{tabular}{llll}
\hline \multirow{2}{*}{ Pod Spot } & \multicolumn{2}{l}{ Neighborhood Pods } & \\
\cline { 2 - 4 } & Caterpillar & Grasshopper & Total Time \\
\hline 1 & $1: 23: 00$ & $2: 32: 00$ & $3: 55: 00$ \\
2 & $3: 00: 00$ & $0: 35: 00$ & $3: 35: 00$ \\
3 & $1: 46: 00$ & $1: 33: 00$ & $3: 19: 00$ \\
4 & $0: 48: 00$ & $1: 18: 00$ & $2: 06: 00$ \\
5 & $0: 10: 00$ & $0: 00: 00$ & $0: 10: 00$ \\
\& Dining Tables & $1: 33: 00$ & $0: 13: 00$ & $1: 46: 00$ \\
Total Time & $8: 40: 00$ & $6: 11: 00$ & \\
\hline
\end{tabular}

The differences in utilization between pod spaces and between floors at Case \#1 raised a number of questions relative to design intensions:

- Is pod space utilization dependent on educators' perceived ownership of the spaces adjacent to their classrooms? As shown above in Figure 14 (refer back to Figure 14) although all classrooms have a pod spot immediate outside their classroom door, only three of the pod spots are not shared for specialist use. These pod spots are also the spaces that experienced the most use. Over the course of the observation period, pod spot spaces 1,2 and 3 were used by over one hour more than pod spot spaces 4 and 5 , and the dining tables. If the teachers adjacent to pod spot spaces 4 and 5 felt they had full ownership over them, and that the pod spot was perhaps considered a part of their 'owned' classroom, would those pod spaces be utilized more (refer back to Table 2)?

- Are educators holding on to the traditional push-in model? One role of the pod spots was to serve specifically as a pull-out space for specialists, while still maintaining a visual connection to the classroom. However, many of the teachers brought the specialists into their classrooms, as opposed to sending students out into the pod spot. One interventionist speculated that the individual educator's comfort level impacted their use of the pod space, "I think it's a comfort level...I think some of it's classroom management, so that they'll feel more free in coming out and using the spaces. They do have a better classroom management than some of the other classrooms that I worked with" (teacher).

- Is perceived time to transition causing educators to favor the pushing-in vs. pulling-out approach - opposite of the actual time it takes? Observation showed that multiple small groups could transition between the pod spot space and the classroom for intervention activities within seconds. The proximity to the classroom of some pod spot spaces resulted in a negligible transition time between 
classroom and pod spot space and is likely comparable to the time it takes students to transition to activities within the classroom. However, there is potential that the perception of transition time from classroom to pod-spot is higher than what was observed. It could be argued that if the perception was that closer pod spots lead to more usage which would equal more, shorter transitions that feel (or actually add up to) more actual time on task than fewer, longer transitions.

The expanded push-in model has shown expected and differences in use. The traditional model had its own set of issues in the pod spots (see Figure 15).
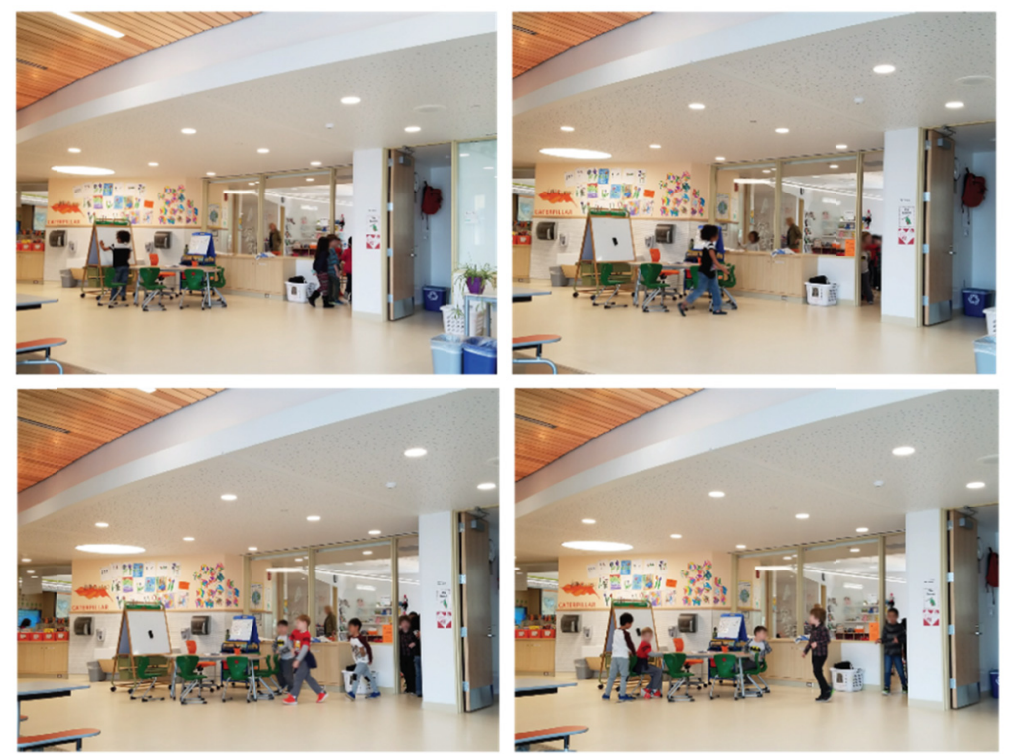

Figure 15. Rapid small group transition between classroom and pod spots

\subsubsection{At Case \#2}

At Case \#2's location, six classrooms shared a singular, large pod space informally divided (see Figure 16). Additionally, the mixed grade levels in that pod group meant no classroom or grade level had ownership of the pod space. As such, multiple groups of different grade levels can do learning within that pod space simultaneously. The use of the pod space must be scheduled - the different teachers must coordinate use of the space among them, or utilize a third pod space outside of the pod (see Figure 16).

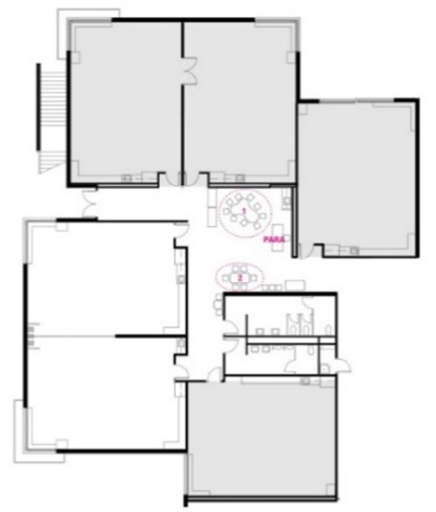

POD SPOTS CASE \#2:

1,2 \& PARA

Figure 16. Pod spot locations for Case \#2, Spots 1,2, and Para shown

Note. Ancillary pod spot is located outside of pod and therefore not shown above. 
The pod space for Case \#2's is much smaller than available at Case \#1's. Case \#2's pod spot space was set up with two tables, stacks of chairs, and one desk for para-professionals. At best, two groups could use the pod space simultaneously, so long as neither one was too loud or disruptive. An additional ancillary area was available around the corner, utilized when the pod area was crowded. This situation occurred on the second day of observations as kindergarten students were working on an art project in one part of the pod spot space while another grade level engaged in a separate small group activity (see Table 4)

Table 4. Total time utilized for small group at Case \#2

\begin{tabular}{lll}
\hline \multirow{2}{*}{ Pod Spot } & \multicolumn{2}{l}{ Total Time Utilized for Small Group at Case \#2 } \\
\cline { 2 - 3 } & Day 1 & Day 2 \\
\hline 1 & $0: 50: 18$ & 0 recorded \\
2 & $0: 31: 02$ & $1: 14: 29$ \\
Ancillary Pod & 0 recorded & $0: 07: 25$ \\
Total Time & $1: 21: 20$ & $1: 21: 54$ \\
\hline
\end{tabular}

These data points indicate the use of the shared pod spot space by the kindergarten classes, not including the use by the first or third grade classes. Increased coordination as a result of shared use of the pod spots could account for the more limited pod spots use by the kindergarten classes at Case \#2.

\section{Discussion of Ethnographic Insights into Pod Use-Case \#1 Only}

A discussion of the ethnographic insights is shared. These data were only collected for the Case \#1 project as this new design was considered the 'control.' Behavioral observations provided quantitative data regarding pod spot space utilization, and the photographic traces' technique was used only at the Case \#1 location to 'dig deeper' to determine if the design intent was being utilized. The findings primarily yielded how the pod spots and overall pod neighborhoods were used, and what adjustments educators made to make them more suitable for themselves. In this descriptive analysis approach, the researchers followed Zeisel's (1984) categories of spatial traces. These included: (a) adaptations of space, (b) displays of self, (c) displays of student and teacher work, and (d) identification and wayfinding.

\section{1 (a) Adaptations of Space}

Of the spatial traces, the most telling in this case study were the Adaptations of Space. Zeisel defines these adaptations as "...changes that users make to an environment so it is better suited to something they want to do" (p. 170). These changes manifest in different ways; some descriptions follow along with descriptive analysis (see Figure 17).

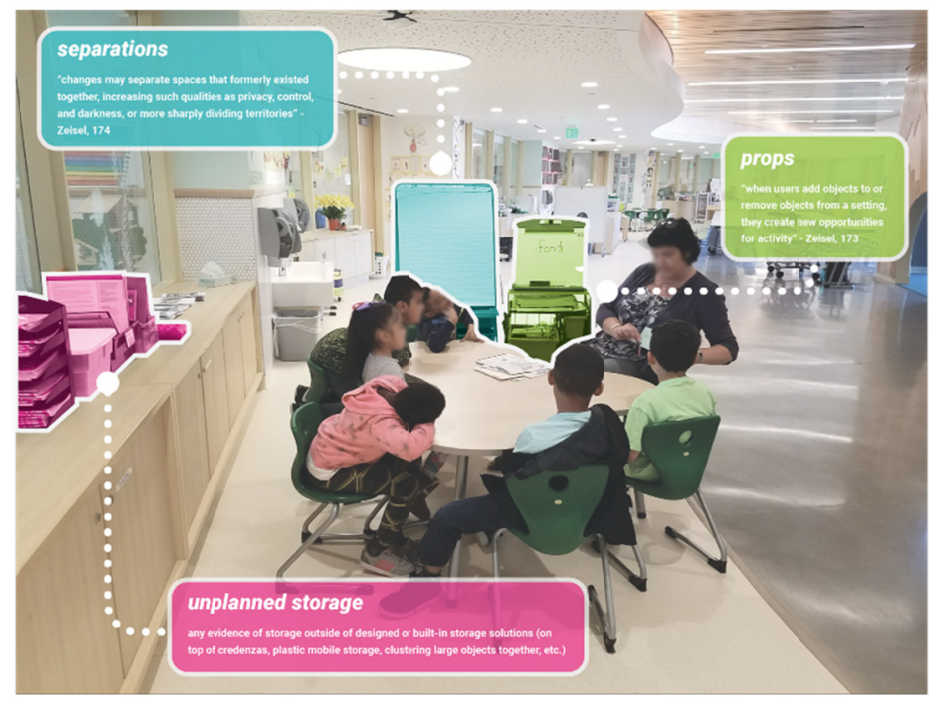

Figures 17. Adaptations of space and definitions for categories

One of the most recorded adaptations at Case \#1 was the use of mobile whiteboards as movable screens for 
visual separation (i.e., 'a prop') - adaptations mitigating distraction. A limit to the efficacy of the pod spot spaces was how open they were. Without any visual or acoustic separation, the design allowed for potentially greater distraction for the kindergartener learning in the pod spot space:

“...if they are out in the pod (and) you're coming by or you've got a student, it's like 'Why do you have THEM?' Why do you have...?' 'What did THEY do wrong?' You know? Things like that, so, it's always like, 'pay attention"'- teacher

The photographic trace analysis corroborated this observation, showing that in most instances, specialists used the mobile dry erase boards to block views to the rest of the pod in order to limit distractions in adjacent pod spots (see Figure 18).
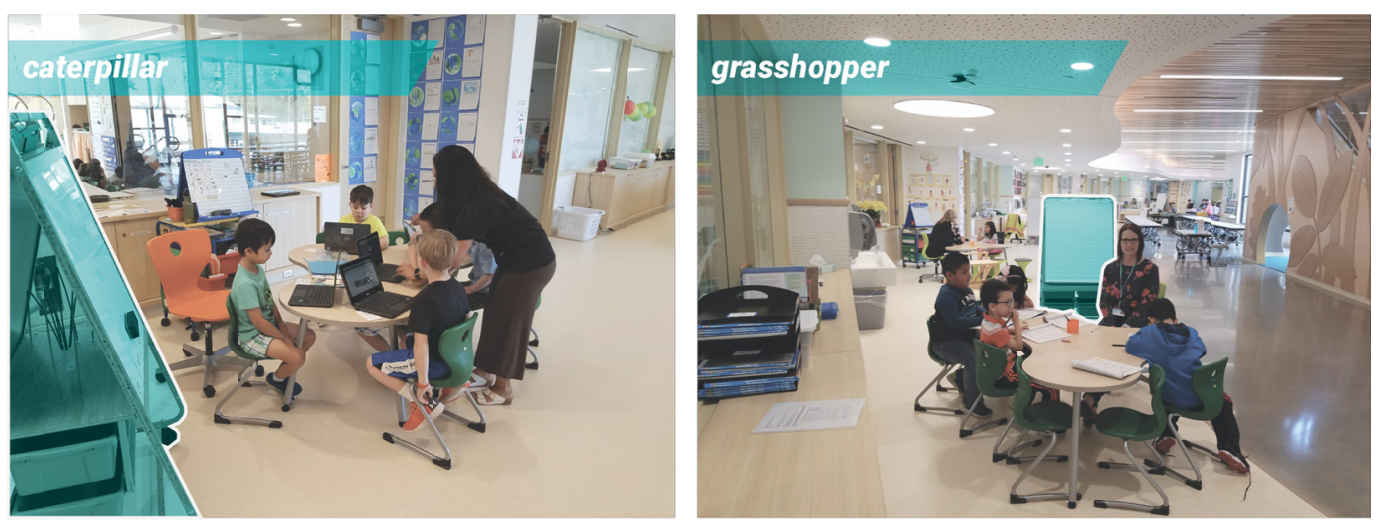

Figure 18. Separations for small-group activities in pods

This strategy appeared to be more successful if adjacent pods spots were engaged in similar or quieter activities. In instances where adjacent pods were engaged in play-to-learn or move-to-learn activities, the lack of acoustic separation between pod spots resulted in some students becoming distracted (see Figure 19).

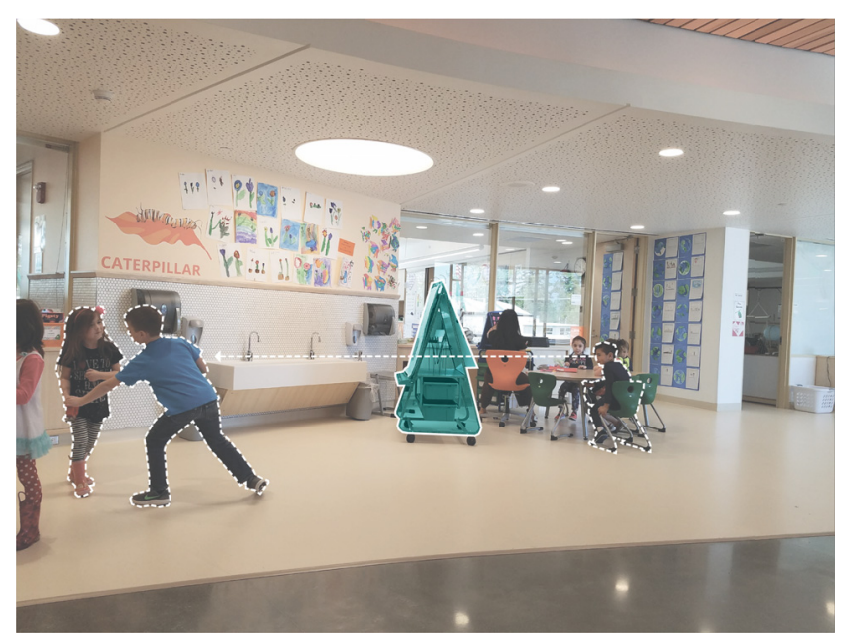

Figure 19. Learner distraction in the pod

The mobile whiteboards were also used as privacy screens at the para-professional desks in both the Grasshopper and Caterpillar pod neighborhoods-adaptations for privacy. One mobile whiteboard appeared in the photographs as a permanent fixture, screening the desks from the pod spot on the other side (see Figure 20). 

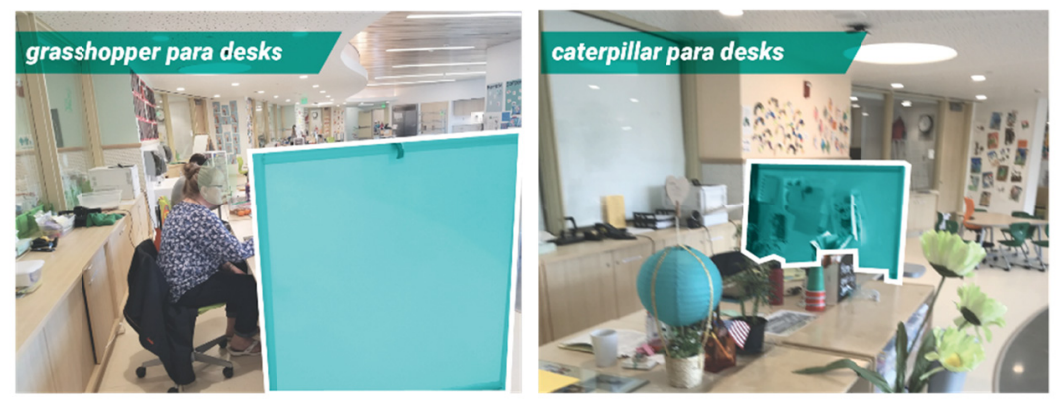

Figure 20. Para-professional's desks at both Grasshopper and Caterpillar neighborhood pods

Unplanned Storage as a category is under adaptation of space. A second very evident adaptation in both the Caterpillar and Grasshopper pod neighborhoods was the proliferation of additional storage solutions, outside of the planned, built-in storage areas (see Figure 21). It is unclear whether the storage solutions provided at Case \#1 are insufficient to store all teaching materials and manipulatives used in the pod spots, or if the campus underutilizes its closed, built-in storage in favor of easier to access, albeit visually distracting, open storage bins atop the built-ins.
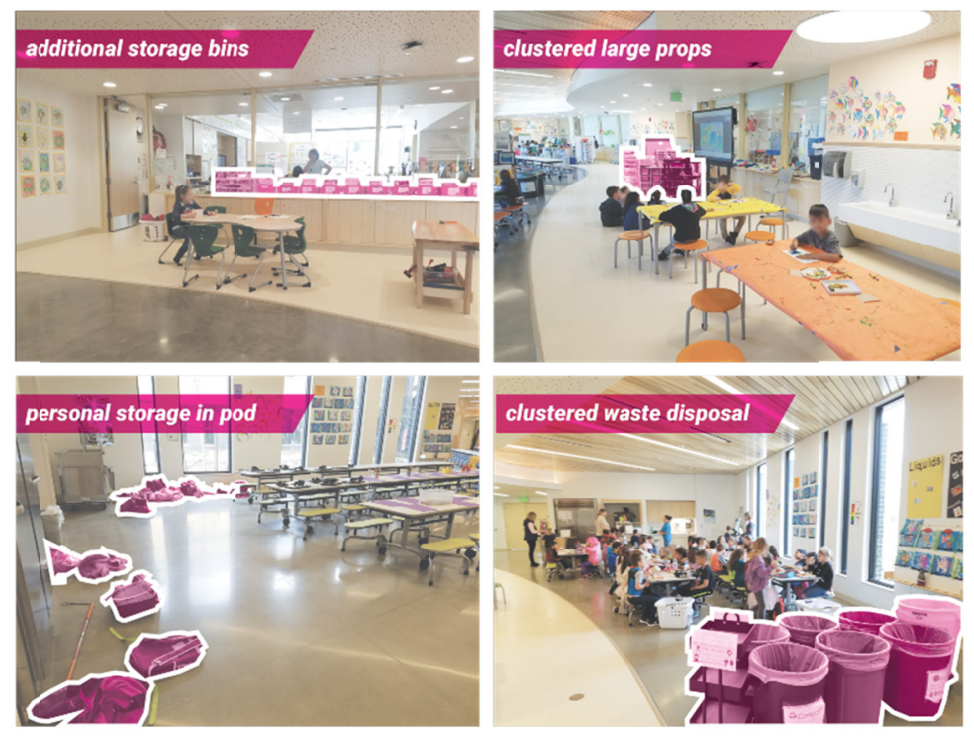

Figure 21. Examples of unplanned storage

Included in this Unplanned Storage category is waste/recycle disposal area (refer back to Figure 21). It is evident that a home for large trash/recycle/compost bins, of which there are many at Case \#1, was not intentionally determined. As a result, these many bins contribute to the visual clutter of the space while additionally acting as a physical barrier to travel freely among the dining table area. Whether this barrier affected the utilization of the dining table area was unclear.

Like the waste/recycle disposal bins, large carts and props did not appear to have a specific home or place to be stored in the pod neighborhoods. For the most part, this situation did not create clutter in the pod spots, except for pod spot 4 and 5. Due to these pod spots' use as a specialist area (i.e., used for art and technology), there were: mobile storage carts, mobile drying racks, light tables, and all manner of other large items clustered together outside of pod spots 4 and 5 (refer back to Figure 21). Not only did this type of clustering contribute to both visual and physical clutter of the space as well as barriers to ease of movement. These solutions also blocked off supervision of that space from elsewhere in the neighborhood.

\subsection{Displays of Self}

Zeisel's (1984) category of spatial traces, Displays of Self, is also very telling in the context of this case study. 
Displays of Self describes: (a) student work and teaching materials, (b) identification, and (c) personalization (see Figure 22).

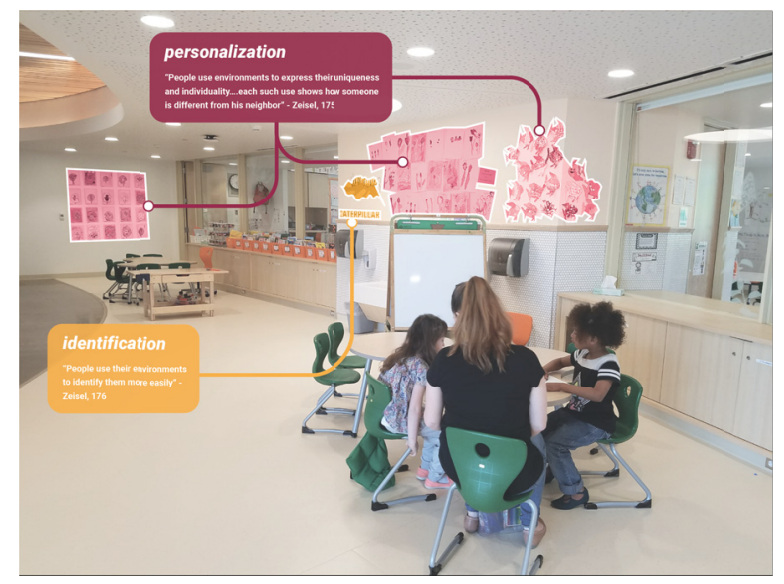

Figure 22. Displays of self and definitions

\subsubsection{Displays of Student Work and Teaching Materials/Personalization}

One of the most common characteristics of school environments is the proliferation of teaching materials and student work displayed in common areas (i.e., personalization). While the pod spots are intended to act as extensions of the classroom environment, they also serve as a gallery for student work. Within the classrooms, wall space is heavily covered with various teaching materials and student work. While the display of student work is important for student confidence and growth (Anonymous, 2010). There is also research available detailing visual complexity concerns (Fisher, Goodwin, \& Seltman, 2014) in the learning environment and its impact on learning outcomes. The observers recorded multiple instances when kindergarteners were distracted by the various visual displays in the pod spots (see Figure 23).

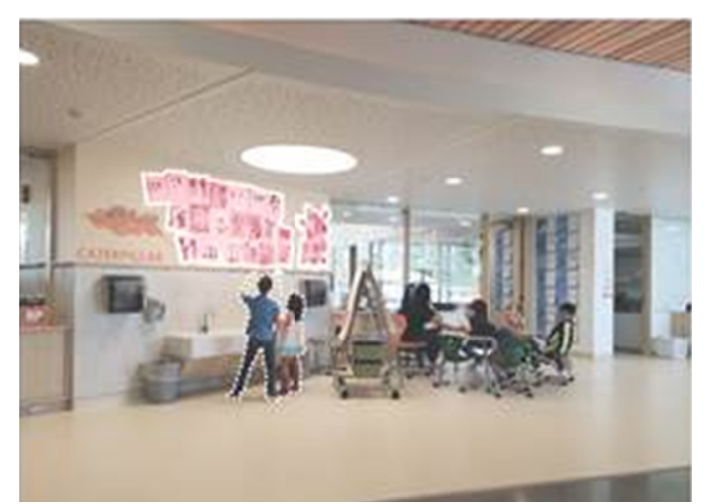

Figure 23. Example of students distracted by display

Although all walls including transparent glass were covered with materials, educators still felt that they needed even more wall space for display: "There's very limited space to post those things in a classroom ...so much of the wall space is windows. From the classroom to the interior pod, and then from the pod to the outside, so you do have to give up functionality and instructional space by having so much glass." - teacher

This typical teacher's viewpoint resulted in the display of teaching materials and student work covering the glass from the classroom into the pod spots. This act reduced the visibility from the classroom into the adjacent pod spot, and also the teachers' ability to passively monitor that spot. By covering the glass, the utilization of the pod spots' supervision is compromised (see Figure 24 and Table 5). 

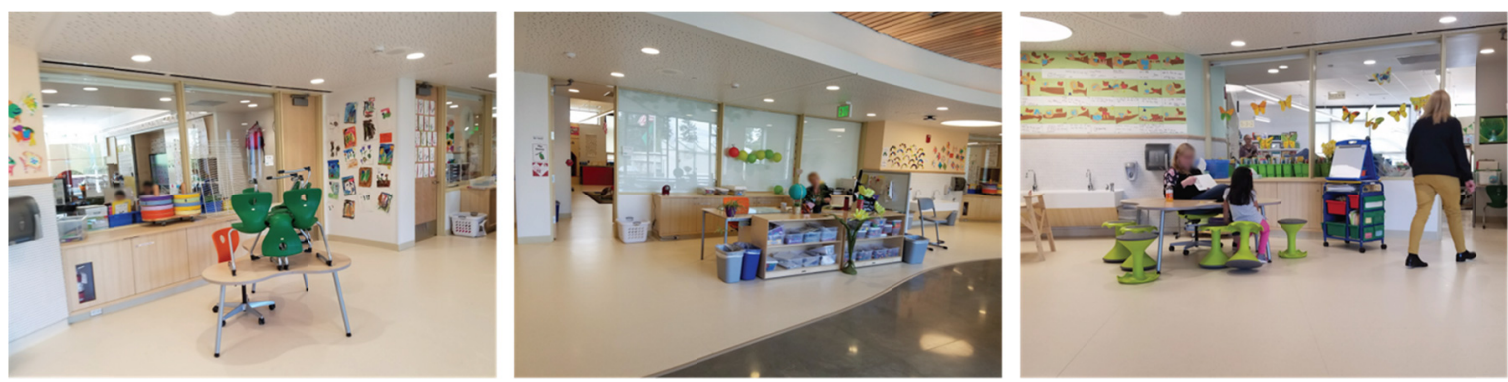

Figure 24. Views into pod covered by displays on glazing/personalization

In the context of this study, the filling of all available vertical wall space is the most prolific form of personalization and is much more impactful, as there are not extraneous decorations in the pod areas.

Table 5. Classroom comparisons of vertical space coverage by floor

\begin{tabular}{llll}
\hline \multicolumn{2}{l}{ Percentage of Vertical Space Coverage } & \\
\hline $\begin{array}{l}\text { Classrooms } \\
\text { Caterpillar }\end{array}$ & Percentage Covered & $\begin{array}{l}\text { Classrooms } \\
\text { Grasshopper }\end{array}$ & Percentage Covered \\
\hline A & $15 \%$ & A & $12 \%$ \\
B & $4 \%$ & B & $24 \%$ \\
C & $100 \%$ (shade drawn) & C & $18 \%$ \\
D & $25 \%$ & D & $15 \%$ \\
E & N/A (researchers unable to tell from & E & $20 \%$ \\
& photographs) & & \\
F & $8 \%$ & F & $45 \%$ \\
\hline
\end{tabular}

\subsubsection{Identification and Wayfinding}

Some instances of the displays on the wall provide guides for the identification of each neighborhood and wayfinding for the kindergarteners. Because each neighborhood's floorplan at Case \#1 was identical, the different visual designations for each may be helpful for kindergarteners to identify his/her own neighborhood and ensure they are in the right place (refer back to Figure 5).

\subsection{RQ.3. What Other Impacts Does a New Neighborhood Pod Design Have on Kindergarten Learners, Educators, and School Staff?}

The interviews of staff and leadership were only administered at Case \#1 and these indicated that there are a multitude of intangible impacts of both the new design model and the $600+$ student kindergarten center typology overall. These findings centered on relationships between students, adults, and between students and adults.

\subsubsection{Relationships}

Relationships between students, adults, and between students and adults are not only difficult to measure in terms of the strength and quality, but also in the impact these relationships might have on the students overall learning experience. It is well established that a key factor in the quality of a learning environment in kindergarten is the quality of the adult - child interactions (Ostrosky \& Jung, n.d.). However, Case \#1 goes beyond these criteria to intentionally create opportunities for an environment improving adult relationships, and allowing them to better serve the children of their school.

Despite the size of the campus, the scale of the neighborhood makes the experience of school much easier for the kindergarteners, and allows them to connect with their peers. In the interviews, one educator agreed that, "...having the six classrooms just be so open to each other, lots of glass, and all the kids eating together for breakfast and lunch, I think just the collaboration is probably the best. And the kids just feel like they're really connected with each other's classes in his/her (neighborhood)."

The typology of a kindergarten center lends itself to a collective focus and drive across the entire campus. Educators felt that with, "...everybody in one building, focused specifically on one age group gives us the best opportunity to grow, to learn, and really improve our work here as a school." All members of staff, leadership, and faculty are focused on the needs of a singular cohort, allowing them to collaborate more easily. 
Additionally, the specific design of the campus fosters collaborative relationships that are not so easily formed on traditionally designed campuses. Although classrooms are still owned, educators have a shared office allowing them to more easily collaborate with their neighbor.

At Case \#1, "...having ... the office together and the doors together, we have that community." In this way, educators felt that "...there's that constant collaboration going on ... whereas, in a traditional classroom you're in a room with yourself and the students and then with the support staff, they're usually in their own offices... with this, we're all together, and we're sharing."

This last finding was particularly true for the specialists teaching technology, art, music, physical education, etc. In a traditional school, specialists own their own spaces and therefore become siloed in their own work around a school. During the interviews, one specialist indicated, "This (meaning the design at Case \#1) is incredible. I've never sat next to a teacher. We were always in our own little island, and never would talk."

Because educators have quick and convenient access to each other through their shared offices and connected classrooms, kindergarteners at Case \#1 have an additional adult that they know and become comfortable with. One teacher felt like she could, “...pop [her] head into [her] teaching partner's classroom and be like, 'Hey.' And the kids know [her] as another adult to talk to. Whereas, [she didn't] feel like [that] in our other schools..."

The new design model also allowed other adults to develop stronger relationships with the kindergarteners. Specialists got to know their students better, since they only work with two neighborhoods, as opposed to a full campus. Additionally, students were able to form better relationships with food service staff. Food service staff served a smaller group of students at one time, allowing them to form better relationships. One food service staff member felt that working at Case \#1, "has been an amazing experience...here you kind of get surrounded with the kids, and they give you a lot of appreciation feedback..." They felt welcomed.

The design of Case \#1 isn't simply about creating a school. It's about creating a sense of community. One educator felt that, "the design really lends itself to a family-kind of community, and...that impact is huge!"

\subsubsection{Transparency}

One of the key design elements at Case \#1 is the use of transparency (glass between the classroom and the pod spot area). The design intent for the transparency was to allow for passive supervision, passive observation, and increased daylight penetration into the pod spot. An educator's immediate reaction might be one of consternation. After just one year, all worries were apparently overcome. According to the campus leader, “... when we first saw this building, I was skeptical. I thought it's so open. It's like a fishbowl, and the kindergarteners are going to spend their whole day looking out into the common pod neighborhood area... and we're just amazed that that doesn't happen!'

Educators and campus leadership are able to passively supervise and evaluate students in the classroom without interrupting lessons: "...I can walk by and kind of evaluate what's happening in the classroom to determine what my next steps are without kids even looking up or getting distracted from their lessons." Additionally, the transparency allows for easy access and communication among educators, "So when you're in that one pod wing together, you're constantly passing by, seeing each other...the person walks by, so you grab them real quick." Higher levels of communication between teachers are aided by the transparency.

After one year of operation transparency looks compromised with every glazed area covered in something — student work, notices, etc. The question then becomes, "Why are educators covering up the glass?" One probable scenario is that they really do not want people viewing into the classroom.

\subsubsection{Staffing and Operations}

Interviews and observations revealed other unexpected outcomes and potential misalignments at Case \#1. Two items in particular are shared, the new dining model and 'points of sale.' The new dining model at Case \#1 worked well to reduce transition travel times and helped students create better relationships with the adults with whom they interact. However, a few operational issues emerged. Two staff individuals rolled the kitchen food carts daily into one end of the neighborhood pod. The set up required a mobile computer station, or 'point of sale,' to $\log$ the finger prints of the students, and due to this log in procedure long lines of students stretched from one end of the pod neighborhood to the other. This line up time decreased the actual amount of time a student had to physically eat. To mitigate this situation, some teachers held their students in the classroom longer, "Since the dining line is really long if all of us go out there at the same time...I say, 'We'll stay in a sing another song or do some activity instead of having them out there waiting in that line. I keep them in my classroom cause I can see the line." 


\section{Misalignments and Design Discoveries}

Misalignments refer to the gaps in design intent and observed occupant use. These gaps can result in unexpected uses of the design, as well as underutilization.

\subsection{Infrastructural Misalignments}

Electrical outlets in the pod spots were located in the baseboard of the built-in storage along the classroom walls. This position selection was likely intended to unclutter the walls and locate them in such a way where it would be less likely for the kindergarteners to play with or harm themselves with the outlets. However, the observers noted that it was often difficult for adults to reach the outlets at this place. As a result, the adults would ask the students to plug things in, resulting in a safety hazard from what was likely intended as a safety feature.

Each neighborhood had sinks to accommodate the use of messy/maker or art activities. An unexpected finding during interviews was a comment that water pressure from the sink faucets are sufficient for washing hands, however the water pressure was insufficient for washing paintbrushes, etc. (see Figure 25).

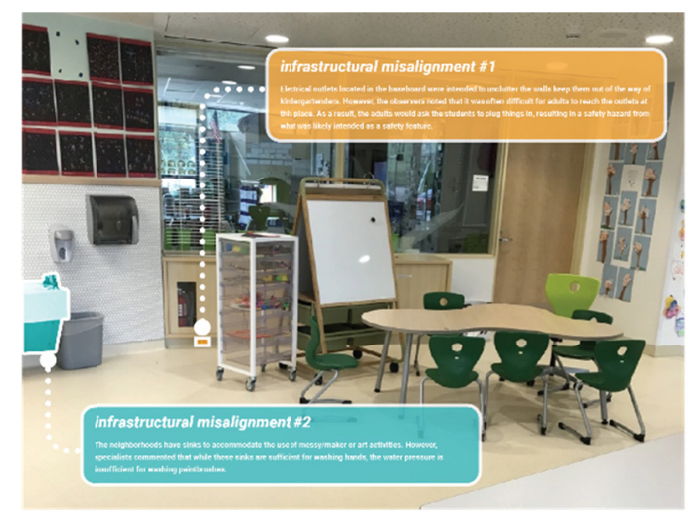

Figure 25. Infrastructural misalignment examples

\subsection{Spatial Misalignments}

\subsubsection{The Nook or 'the Nest'}

Both first floor neighborhoods had a reading nook built underneath the stairs. It's whimsical and inviting with carpeted flooring, a window to the outdoors, and an entry portal that is kindergartener-sized. However, observers noted this space was used in a very limited capacity, and for one key reason: supervision. The only time observers saw the space being used was when an educator took a group inside to do a reading activity - kindergarteners did not use that space by themselves. The observers speculated that this lack of use is likely because educators are unable to supervise students inside the Nook/Nest without being inside with them (see Figure 26).

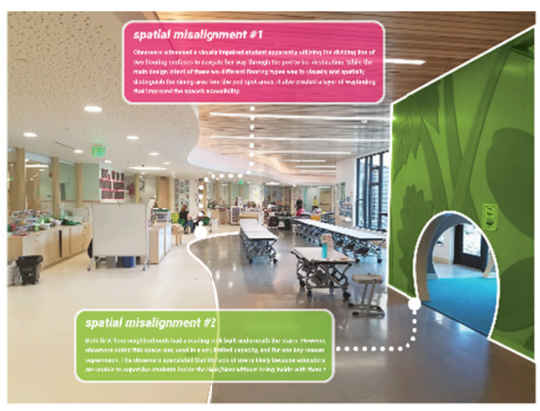

Figure 26. Spatial misalignments 


\subsubsection{Pod Floors and Navigation}

An unexpected design discovery was the use of the two different types of flooring in the neighborhoods (refer back Figure 26) appeared to provide an added navigational aid for a visually impaired student. One observer saw this particular student navigate the neighborhood pod by tapping her cane across the boundaries of the two floor surfaces each time she went from one end of the neighborhood to the other, and she did so multiple times in the course of a day.

While the design intent of providing two different flooring types was to visually and spatially distinguish the dining area from the pod spot areas, it also created a layer of wayfinding that improved the space's accessibility.

\section{Limitations}

A Research Case Study is an opportunity to more fully understand a particular design or model, post-occupancy. The Human-centered Research Design's protocol is a rigorous social science set of mixed-method procedures using multiple types of techniques to gather data limiting research bias. Even so, two days of behavioral observation does not allow for a full understanding of any model. The comparison of one 'traditional' model to a 'new' one is important, however, the convenience sample models are not easily compared on a one-to-one basis and any findings have to be considered under these conditions.

\section{Next Steps}

A new design solution was developed as a result of responding to a new governing mandate. The initial hypothesis was 'proven,' and more questions raised from what was revealed through the research observations from the design intent to the actual use of the spaces. Specific items needing further investigation include the connection between visual 'noise' and distraction and the aforementioned questions presented regarding educator use of ancillary spaces, such as the pod spots (e.g., educator perception and its impact on use and the tendency to stick to traditional models). Professional development may be required to understand how these new space types (pod spots) can be affectively utilized.

\section{Conclusions}

The original design hypothesis was tested along with the design goal of reducing transition travel times as a result of the new 'expanded push-in' design; both were successfully met. Reduced transition travel time equaled more learning time recaptured, and a higher utilization of the built square footage. More staffing was required for the implementation of new dining experience. The new physical design functioned well, but there is always room for improvement. Relationships and student outcomes perceived to be improved with respondents comparing this new place to more traditional design models.

As in any on site case study, a lot of data is generated. The findings were categorized into the following areas: (a) transition time, (b) use of the pods spots, (c) impacts of the new design model, and (d) some side notes that might be of interest. What do these findings mean for others?

\subsection{What Does This Mean for School Leaders?}

Often one doesn't have control over the design process for a campus and the distances students are expected to travel. However, there is often opportunities to select where in the building different age groups will sit, develop their abilities, and the impact this opportunity has on the time it will take to travel. When designing a new campus, consider opportunities for shortening the distances all students especially the younger ones have to travel. Planning the school's schedule so that whole class destinations are grouped together (such as dining, leading in to an adjacent recess) could also assist in reducing the amount of transition time.

\subsection{What Does This Mean for Designers of Schools?}

The development of a one-age cohort is very different. Perhaps the design using the ancient Nautilus's proportions actually encourages a new developmental model for engaging students and educators in a more connected learning process. The new 'expanded push-in model' clearly had advantages over the 'traditional one' The organizations of space types for particular activities with the goal to reduce student transition times, and the generation of neighborhoods supports what is know about how people develop a sense of belonging and therefore adding to the motivation to become more engaged in one's learning process.

\section{Disclosure}

Two of the researchers are members of the architectural firm that designed Case \#1, but were not part of the design team. 


\section{Acknowledgement}

With thanks to our sample respondents for allowing us to observe and compare, and to DLR Group for funding the comparative study project. A further thank you goes to Niyati Gandi for her work in decoding the behavioral observation data.

\section{References}

Anonymous. (n.d.). Child development and early learning. Why it is important to share and act on this information. In Facts for Life (4th ed.). Retrieved August 17, 2019, from http://www.factsforlifeglobal.org/03/

Anonymous. (n.d.).

Retrieved from https://www.dlrgroup.com/work/early-learning-kindergarten-center-at-fairmount/

Anonymous. (2010). Displaying student work. Responsive Classrooms. Retrieved from https://www.responsiveclassroom.org/displaying-student-work/

Anonymous. (2016/January). Full-day kindergarten professional development. Washington State Full Day Kindergarten Guide, 2(2), 27. WA: Superintendent of Public Instruction. Retrieved from https://www.k12.wa.us/sites/default/files/public/bulletinsmemos/memos2016/M034-16Attach1.pdf

Desautels, L. (2016). Creating trauma informed instruction-schools. The heart of teaching and learning. A Presentation by Dr. Lori Desautels at Marian University Preview: Schools as Ecological System. Retrieved October 10, 2019, from $\mathrm{http} / /$ revelationsineducation.com/creating-trauma-informed-instruction-schoolsthe-heart-of-teaching-and-le arning/

Fisher, A. V., Godwin, K. E., \& Seltman, H. (2014). Visual environment, attention allocation, and learning in young children: When too much of a good thing may be bad. Psychological Science, 25(7), 1-10. https://doi.org/10.1177/0956797614533801

French, J., Scott-Webber, L., Ferking, T., \& Fulton, D. (2015). Early childhood space design: Nurturing every student's potential (p. 20). EdSpaces Conference. LA: Education Market Association.

Hanington, B. M. (2010). Relevant and rigorous: Human-centered research and design education. Massachusetts Institute of Technology Design Issues, 26(3), 18-26. https://doi.org/10.1162/DESI_a_00026

Jensen, E. (2005). Teaching with the brain in mind (p. 1). Association for Supervision and Curriculum Development (ASCD).

Johnson, B. R., Onwuegbuzie, A. J., \& Turner, L. A. (2007). Toward a definition of mixed methods research. Journal of Mixed Methods Research, 1, 112-133. https://doi.org/10.1177/1558689806298224

Ostrosky, M. M., \& Jung, E. Y. (n.d.). Building positive teacher-child relationships. What Works Briefs. DC: The Center on the Social and Emotional Foundations for Early Learning. Retrieved from http://csefel.vanderbilt.edu/briefs/wwb12.pdf

Reykdal, C. (n.d.). Early learning in Washington State. State-funded full-day kindergarten. WA: Office of $\begin{array}{lllll}\text { Superintendent } & \text { of Public } & \text { Instruction. } & \text { Retrieved }\end{array}$ https://www.k12.wa.us/student-success/support-programs/early-learning-washington-state

Scott-Webber, L. (2011, June). Education design: what it takes to really understand what we are designing for in a classroom setting. NeoCon World's Trade Fair 2007. MI. Retrieved from http://www.neocon.com/education/seminars/conference-proceedings/W313.

Taylor, S. (2011). Characteristics of great kindergarten classrooms. Retrieved September 15, 2019 from https://www.scholastic.com/teachers/blog-posts/sharon-taylor/characteristics-great-kindergarten-classrooms l

Zeisel, J. (1984). Inquiry by design: Tools for environment-behavior research. Cambridge, New York, NY, USA: Cambridge University Press. https://doi.org/10.2307/2066778

\section{Copyrights}

Copyright for this article is retained by the author, with first publication rights granted to the journal.

This is an open-access article distributed under the terms and conditions of the Creative Commons Attribution license (http://creativecommons.org/licenses/by/4.0/). 